\title{
Preference stability along time: The time cohesiveness measure
}

\author{
T. González-Artega • R. de Andrés Calle · M. Peral
}

Received: date / Accepted: date

\begin{abstract}
This work introduces a non-traditional perspective about the problem of measuring the stability of agents' preferences. Specifically, the cohesiveness of preferences at different moments of time is explored under the assumption of considering dichotomous evaluations. The general concept of time cohesiveness measure is introduced as well as a particular formulation based on the consideration of any two successive moments of time, the sequential time cohesiveness measure. Moreover, some properties of the novel measure are also provided. Finally, and in order to emphasize the adaptability of our proposal to real situations, a factual case of study about Clinical Decision Making is presented. Concretely, the study of preference stability for life-sustaining treatments of patients with advanced cancer at end of life is analysed. The research considers patients who express their opinions on three life-sustaining treatments at four consecutive periods of time. The novel measure provides information of patients preference stability along time and considers the possibility of cancer metastases.
\end{abstract}

T. González-Artega

BORDA and PRESAD Research Groups and

Multidisciplinary Institute of Enterprise (IME),

University of Valladolid, E47011 Valladolid, Spain

E-mail: teresag@eio.uva.es

R. de Andrés Calle

BORDA Research Unit, PRESAD Research Group and

Multidisciplinary Institute of Enterprise (IME),

University of Salamanca, E37007 Salamanca, Spain

E-mail: rocioac@usal.es

M. Peral

Centro de Atención Primaria de Laguna de Duero,

E47140, Valladolid, Spain

E-mail: mperalh@saludcastillayleon.es
Keywords Time cohesiveness measure - Dichotomous opinions · Preference stability · Patients' preferences

\section{Introduction}

Intertemporal decision making is an important scientific area and it has been obtaining attention from several research fields such as Economics, Health Economics, Social Choice, Psychology, Marketing, Decision Analysis, Neuroscience, and so on.

One of the main topics of this area is the study of preference stability that is often defined like the measurement of the choice consistency among options along time [8], [20], [28]. Traditionally, preferences have usually been considered permanent by theory [23], although there are also different studies to check if they are constant over time [4], [7], [11], [26]. Related to empirical literature on preference stability, most studies use small samples in short time periods and they are focused on a specific type of preferences, the risk preferences [27]. Recently, there has been an increment of works about time preference [12], [22], [24], while there are few contributions that study the stability of social preference [10].

From another point of view, a growing number of studies considers changes in preferences as a result of shocks such as illness, civil wars, natural disasters, etc. $[9],[15],[21],[25]$.

The research to date has tend to explore preference stability by means of statistical approaches: from basic methods like descriptive analysis and multiple regression [22], [28] to more elaborate procedures like hierarchical generalized linear modelling [8] and others [29].

In order to enhance the preference stability topic, the aim of this contribution is to develop a new tool 
capable of measuring preference stability from a nontraditional perspective. For this purpose, the notion of preference stability is considered in the same vein that the notion of cohesiveness. This seems natural because the measurement of preference stability resembles the notion of measurement of cohesiveness over time, in the sense that the maximum value captures the notion of full stability, that is unanimity along time, while the minimum value captures the notion of total lack of stability, that is, total disagreement along time.

The cohesiveness or consensus measurement has been dealt in the Social Choice literature from Bosch's seminal work [6]. Subsequently, Alcalde-Unzu and Vorsatz [1], Alcantud et al. [2] and García-Lapresta and Pérez-Román [14] introduced several classes of consensus measures based on distances for ordinal information. Additionally, several studies related to consensus problem deal also with cardinal information like the approaches proposed by González-Arteaga et al. [16], González-Pachón and Romero [17], González-Pachón et al. [18], Herrera-Viedma et al. [19], and so on. From another point of view, Alcantud, de Andrés Calle and Cascón [3] introduced a cohesiveness measure when opinions are dichotomous.

Taking into account the previous contributions on preference stability and cohesiveness measure, this paper is focused on an inter-temporal decision making problem where a set of agents express their opinions on an alternative along different moments of time. To be precise, agents have to approve or disapprove the alternative under study at diverse point of time. Thus, the paper objective is to determine how much stability or cohesiveness agents' opinions conveys to the group on the alternative along time. In order to measure such stability, a new general approach is defined, the time cohesiveness measure. Following the Social Choice tradition, this measurement takes values in the unit interval considering value 1 full stability and value 0 total lack of stability. Moreover, an specific formulation of the time cohesiveness measure is introduced, the sequential time cohesiveness measure as well as a study of its analytic properties. Under this approach, the stability of preferences is understood like the probability that for a randomly chosen moment of time, two randomly chosen agents have the same opinion at such a time and its consecutive.

Furthermore, the measurement proposed is put in practice in a real case of study to emphasise its applicability. In particular, the stability of preferences for life-sustaining treatments in terminally cancer patients' last year of life is analysed.
The paper is structured as follows. It has been divided into three parts. The first part, Section 2, introduces our proposal to measure preference stability: the time cohesiveness measure. Moreover, an specific type of this measure, the sequential time cohesiveness measure, is presented as well as its properties. The second part, Section 3, includes an application of the novel approach to a real case of study. Finally, some concluding remarks are provided.

\section{A new tool to measure preference stability: The time cohesiveness measure}

This section is devoted to introduce some notation as well as our proposal of measurement of preference stability, namely, the time cohesiveness measure. Then, an specific formulation, the sequential time cohesiveness measure, is defined and its properties are examined.

\subsection{Notation}

Let $\mathbf{N}=\{1,2, \ldots, N\}$ a set of agents or experts. Agents express their opinions on an alternative, $x$, at different time moments $\mathbf{T}=\left\{t_{1}, \ldots, t_{T}\right\}$ by means of dichotomous opinions.

From now on, the notation used to formalize theses assessments is the following:

Definition 1 A time preference profile of a set of agents $\mathbf{N}$ on an alternative $x$ at $T$ different time moments is an $N \times T$ matrix

$$
\mathbf{P}=\left(\begin{array}{ccc}
P_{1 t_{1}} & \ldots & P_{1 t_{T}} \\
\vdots & \ddots & \vdots \\
P_{N t_{1}} & \ldots & P_{N t_{T}}
\end{array}\right)_{N \times T}
$$

where $P_{i t_{j}}$ is the opinion of the agent $i$ over alternative $x$ at $t_{j}$ moment, in the sense

$P_{i t_{j}}=\left\{\begin{array}{l}1 \text { if agent } i \text { approves } x \text { at the } t_{j} \text { time, } \\ 0 \text { otherwise. }\end{array}\right.$

Let $\mathbb{P}_{N \times T}$ denote the set of all such $N \times T$ matrices. For simplicity of notation, $(1)_{N \times T}$ is the $N \times T$ matrix whose cells are universally equal to 1 and $(0)_{N \times T}$ is the $N \times T$ matrix whose cells are universally equal to 0 .

A time preference profile $\mathbf{P}$ is unanimous if alternative $x$ is approved (resp. disapproved) over $\mathbf{T}$ by all agents. In matrix terms, if the time preference profile $\mathbf{P} \in \mathbb{P}_{N \times T}$ is constant, $\mathbf{P}=(1)_{N \times T}$ (resp. $\left.\mathbf{P}=(0)_{N \times T}\right)$. 
Any permutation $\sigma$ of the agents $\{1,2, \ldots, N\}$ determines a time preference profile $\mathbf{P}^{\sigma}$ by permutation of the rows of $\mathbf{P}$, that is, row $i$ of the profile $\mathbf{P}^{\sigma}$ is row $\sigma(i)$ of the profile $\mathbf{P}$.

For each time preference profile $\mathbf{P}, \mathbf{P}_{S}$ is the restriction to a subset of agents, an agent-subprofile on the agents in $S \subseteq \mathbf{N}$, and it emerges from selecting the rows of $\mathbf{P}$ that are associated with the respective agents in $S$.

For each time preference profile $\mathbf{P}, \mathbf{P}^{I}$ is the restriction to a subset of consecutive moments of time, timesubprofile on the moments of time in $I \subseteq \mathbf{T}$, and it emerges from selecting consecutive columns of $\mathbf{P}$ that are associated with the respective moments of time in I. Any partition $\left\{I_{1}, \ldots, I_{p}\right\}$ of $\mathbf{P}$ generates a decomposition of $\mathbf{P}$ into time-subprofiles $\mathbf{P}^{I_{1}}, \ldots, \mathbf{P}^{I_{p}}$ where $\mathbf{P}^{I_{1}} \cup \ldots \cup \mathbf{P}^{I_{p}}=\mathbf{P}$.

An extension of a time preference profile $\mathbf{P}$ of a group of agents $\mathbf{N}$ at $\mathbf{T}=\left\{t_{1}, \ldots, t_{T}\right\}$ is a time preference profile $\overline{\mathbf{P}}$ at $\overline{\mathbf{T}}=\left\{t_{1}, \ldots, t_{T}, t_{T+1}, \ldots, t_{T+q}\right\}$ such that the restriction of $\overline{\mathbf{P}}$ to the first $T$ moments of time of $\overline{\mathbf{T}}$ coincides with $\mathbf{P}$.

A replication of a time preference profile $\mathbf{P}$ of a group of agents $\mathbf{N}$ on alternative $x$ is the time preference profile $\mathbf{P} \uplus \mathbf{P} \in \mathbb{P}_{2 N \times T}$ obtained by duplicating each row of $\mathbf{P}$, in the sense that rows $r$ and $N+r$ of $\mathbf{P} \uplus \mathbf{P}$ are row $r$ of $\mathbf{P}$, for each $r=1, \ldots, N$.

For each time preference profile $\mathbf{P}$ on alternative $x$, $n_{0}^{t_{j}}$ denotes the number of agents that disapprove $x$ at the $t_{j}$ moment of time, and $n_{1}^{t_{j}}$ denotes the number of agents that approve alternative $x$ at the $t_{j}$ moment of time. Therefore, $N=n_{0}^{t_{j}}+n_{1}^{t_{j}}$ for each $t_{j} \in \mathbf{T}$.

In addition, $n_{0,0}^{t_{j}, t_{j+1}}$ denotes the number of agents that disapprove alternative $x$ at $t_{j}$ and keep their opinion at the following point of time $t_{j+1}$. Similarly, $n_{1,1}^{t_{j}, t_{j+1}}$ denotes the number of agents that approve alternative $x$ at $t_{j}$ and keep their opinion at the following point of time $t_{j+1}$.

In this way, $n_{0,1}^{t_{j}, t_{j+1}}$ is the number of agents that disapprove alternative $x$ at $t_{j}$ but change their opinion at $t_{j+1}$, and $n_{1,0}^{t_{j}, t_{j+1}}$ is the number of agents that approve alternative $k$ at $t_{j}$ but change their opinion at $t_{j+1}$. For each $t_{j} \in \mathbf{T}, n_{0}^{t_{j}}=n_{0,0}^{t_{j}, t_{j+1}}+n_{0,1}^{t_{j}, t_{j+1}}$ and likewise $n_{1}^{t_{j}}=n_{1,1}^{t_{j}, t_{j+1}}+n_{1,0}^{t_{j}, t_{j+1}}$. See Table 1 for improving understanding.

For the purpose of clarifying the use of the previous notation, the following illustrative example is introduced.

\begin{tabular}{l|rr|r}
$t_{j}$ & No & Yes & \\
\hline No & $n_{j+1}^{t_{j}, t_{j+1}}$ & $n_{0,1}^{t_{j}, t_{j+1}}$ & $n_{0}^{t_{j}}$ \\
Yes & $n_{1,0}^{t_{j}, t_{j+1}}$ & $n_{1,1}^{t_{j}, t_{j+1}}$ & $n_{1}^{t_{j}}$ \\
\hline & $n_{0}^{t_{j+1}}$ & $n_{1}^{t_{j+1}}$ & $N$
\end{tabular}

Table 1 Notation summary table

Example 1 Let $\mathbf{N}=\{1,2, \ldots, 10\}$ be a set of ten agents that express their opinions on alternative $x$ along four consecutive moments of time $\mathbf{T}=\left\{t_{1}, t_{2}, t_{3}, t_{4}\right\}$. Their time preference profile is:

$$
\mathbf{P}=\left(\begin{array}{ccc}
P_{1 t_{1}} & \ldots & P_{1 t_{4}} \\
\vdots & \ddots & \vdots \\
P_{10 t_{1}} & \ldots & P_{10 t_{4}}
\end{array}\right)_{10 \times 4}=\left(\begin{array}{cccc}
1 & 1 & 1 & 0 \\
0 & 1 & 1 & 0 \\
0 & 1 & 0 & 0 \\
0 & 1 & 0 & 1 \\
1 & 0 & 1 & 1 \\
1 & 1 & 1 & 1 \\
0 & 0 & 0 & 0 \\
0 & 0 & 1 & 1 \\
0 & 1 & 1 & 0 \\
0 & 1 & 0 & 1
\end{array}\right)
$$

This time preference profile can be summarized in a table containing the number of agents who approve or disapprove alternative $x$ at each moment of time $t_{j}$ as well as the number of agents that keep or change their opinion during consecutive time moments (see Table 2).

2.2 New approach to measure preference stability:

Definition and properties

Influenced by Bosch's consensus approach [6], our proposal of cohesivenesses measure along time is introduced below.

Definition 2 A time cohesiveness measure for a group of agents $\mathbf{N}=\{1, \ldots, N\}$ on an alternative $x$ is a mapping

$$
\tau: \mathbb{P}_{N \times T} \rightarrow[0,1]
$$

that assigns a number $\tau(\mathbf{P}) \in[0,1]$ to each time preference profile $\mathbf{P}$, with the properties:

i) $\tau(\mathbf{P})=1$ if and only if $\mathbf{P}$ is unanimous (full stability).

ii) $\tau\left(\mathbf{P}^{\sigma}\right)=\tau(\mathbf{P})$ for each permutation $\sigma$ of the agents and $\mathbf{P} \in \mathbb{P}_{N \times T}$ (anonymity).

A time cohesiveness measure is a collection of time cohesiveness measures for each group of agents $\mathbf{N}$. 


\begin{tabular}{l|rr|r}
\multicolumn{1}{c|}{$t_{1}$} & No & Yes & \\
\hline No & $n_{0,0}^{t_{1}, t_{2}}=2$ & $n_{0,1}^{t_{1}, t_{2}}=5$ & $n_{0}^{t_{1}}=7$ \\
Yes & $n_{1,0}^{t_{1}, t_{2}}=1$ & $n_{1,1}^{t_{1}, t_{2}}=2$ & $n_{1}^{t_{1}}=3$ \\
\hline & $n_{0}^{t_{2}}=3$ & $n_{1}^{t_{2}}=7$ & $N=10$
\end{tabular}

\begin{tabular}{l|rr|l} 
& No & Yes & \\
\hline$t_{2}$ & $t_{3}$ & & \\
No & $n_{0,0}^{t_{2}, t_{3}}=1$ & $n_{0,1}^{t_{2}, t_{3}}=2$ & $n_{0}^{t_{2}}=3$ \\
Yes & $n_{1,0}^{t_{2}, t_{3}}=3$ & $n_{1,1}^{t_{2}, t_{3}}=4$ & $n_{1}^{t_{2}}=7$ \\
\hline & $n_{0}^{t_{3}}=4$ & $n_{1}^{t_{3}}=6$ & $N=10$
\end{tabular}

\begin{tabular}{l|rr|l} 
& No & Yes & \\
\hline$t_{3}$ & $t_{4}$ & $n_{0,1}^{t_{3}, t_{4}}=2$ & $n_{0}^{t_{3}}=4$ \\
No & $n_{0,0}^{t_{3}, t_{4}}=2$ & $n_{1,1}^{t_{3}, t_{4}}=3$ & $n_{1}^{t_{3}}=6$ \\
\hline Yes & $n_{1,0}^{t_{3}, t_{4}}=3$ & $n_{1}^{t_{4}}=5$ & $N=10$
\end{tabular}

Table 2 Notation summary table for Example 1

Our proposal in contrast to Bosch's contribution does not require neutrality property, time moments can not be exchanged, due to the fact that time order is an essential aspect to measure the stability of preferences.

Now a particular time cohesiveness measure is introduced. Formally:

Definition 3 The sequential time cohesiveness measure for a group of agents $\mathbf{N}=\{1, \ldots, N\}$ on an alternative $x$ is the mapping $\tau_{S}: \mathbb{P}_{N \times T} \rightarrow[0,1]$ given by

$$
\begin{aligned}
\tau_{S}(\mathbf{P}) & = \\
& =\frac{1}{T-1} \cdot \frac{\sum_{j=1}^{j=T-1} n_{0,0}^{t_{j}, t_{j+1}} \cdot\left(n_{0,0}^{t_{j}, t_{j+1}}-1\right)}{N(N-1)} \\
& +\frac{1}{T-1} \cdot \frac{\sum_{j=1}^{j=T-1} n_{1,1}^{t_{j}, t_{j+1}} \cdot\left(n_{1,1}^{t_{j}, t_{j+1}}-1\right)}{N(N-1)}
\end{aligned}
$$

Intuitively, it measures the probability that for a randomly chosen moment of time, two randomly chosen agents of a group have the same opinion upon an alternative at the moment of time selected and its consecutive.

It is easy to check that Definition 3 provides a time cohesiveness measure.
Hereunder, some desirable properties of the sequential cohesiveness measure are defined and proved.

\section{Properties}

Reversal invariance: This property shows that the main aspect of the time sequential cohesiveness measure is the stability of agents' opinions more than an specific value. If the 0 's are changed for 1 's and vice verse, then the sequential time cohesiveness measure reminds equal. Formally:

Let $\mathbf{P}^{c}$ be the complementary time preference profile of $\mathbf{P}$ defined by $\mathbf{P}^{c}=(1)_{N \times T}-\mathbf{P}$. If $\tau_{S}$ verifies reversal invariance then $\tau_{S}\left(\mathbf{P}^{c}\right)=\tau_{S}(\mathbf{P})$.

Proof Agents' opinions at $t_{j}, t_{j+1} \in \mathbf{T}$ do not change in $\mathbf{P}$ and $\mathbf{P}^{c}$, then $\tau_{S}$ does not change. That is, those agents whose opinions coincide at $t_{j}$ and $t_{j+1}$ in $\mathbf{P}$ have also coincident opinions at $t_{j}$ and $t_{j+1}$ in $\mathbf{P}^{c}$ although those opinions are different than in $\mathbf{P}$. Taking into account the Definition $3, \tau_{S}$ does not change.

Time-reducibility: It means that the stability of a time preference profile is the average of the time cohesiveness measures of all its consecutive timesubprofiles of two consecutive moments of time. Formally:

Let $\mathbf{P} \in \mathbb{P}_{N \times T}$ be a time preference profile. We say that $\tau_{S}$ verifies time-reducibility if

$$
\tau_{S}(\mathbf{P})=\frac{1}{T-1} \sum_{j=1}^{T-1} \tau_{S}\left(\mathbf{P}^{I_{j, j+1}}\right)
$$

where $\mathbf{P}^{I_{j, j+1}} \in \mathbb{P}_{N \times 2}$ is the time-subprofile of $\mathbf{P}$ containing the columns corresponding to times $t_{j}$ and $t_{j+1}$.

Proof It is straightforward from the Definition 3 since

$$
\begin{aligned}
\tau_{S}\left(\mathbf{P}^{I_{j, j+1}}\right) & = \\
& =\frac{n_{0,0}^{t_{j}, t_{j+1}}\left(n_{0,0}^{t_{j}, t_{j+1}}-1\right)}{N(N-1)} \\
& +\frac{n_{1,1}^{t_{j}, t_{j+1}}\left(n_{1,1}^{t_{j}, t_{j+1}}-1\right)}{N(N-1)}
\end{aligned}
$$


Replication monotonicity: When a non-unanimous time preference profile is replicated, its sequential time cohesiveness measure increases. Formally:

Let $\mathbf{P} \in \mathbb{P}_{N \times T}$ be a non unanimous time preference profile then

$$
\tau_{S}(\mathbf{P} \uplus \mathbf{P})>\tau_{S}(\mathbf{P})
$$

Proof Using time-reducibility is enough to prove this property for only two moments of time. Consider $\mathbf{P}^{I_{j, j+1}} \in \mathbb{P}_{N \times 2}$ a time-subprofile for $t_{j}$ and $t_{j+1}$.

$$
\begin{aligned}
& \tau_{S}\left(\mathbf{P}^{I_{j, j+1}}\right)= \\
& =\frac{n_{0,0}^{t_{j}, t_{j+1}}\left(n_{0,0}^{t_{j}, t_{j+1}}-1\right)}{N(N-1)} \\
& +\frac{n_{1,1}^{t_{j}, t_{j+1}}\left(n_{1,1}^{t_{j}, t_{j+1}}-1\right)}{N(N-1)} \\
& \tau_{S}\left(\mathbf{P}^{I_{j, j+1}} \uplus \mathbf{P}^{I_{j, j+1}}\right)= \\
& =\frac{2 n_{0,0}^{t_{j}, t_{j+1}}\left(2 n_{0,0}^{t_{j}, t_{j+1}}-1\right)}{2 N(2 N-1)} \\
& +\frac{2 n_{1,1}^{t_{j}, t_{j+1}}\left(2 n_{1,1}^{t_{j}, t_{j+1}}-1\right)}{2 N(2 N-1)}
\end{aligned}
$$

It is enough that $\frac{2 z-1}{2 N-1}>\frac{z-1}{N-1}$ for each natural number $z \in \mathbb{N}$ with $z<N$. And this is easily checked.

In addition, for an unanimous time preference profile $\mathbf{P} \in \mathbb{P}_{N \times T}$, by Definition $3, \tau_{S}$ verifies

$$
\tau_{S}(\mathbf{P} \uplus \mathbf{P})=\tau_{S}(\mathbf{P})=1
$$

Minimum time stability: If all agents express their opinions at a moment of time and change their opinions at the next moment of time, that is, all agents change their opinions along two successive moments of time, then the sequential time cohesiveness measure takes a zero value. It also happens when there are at most two agents that keep their opinion at two consecutive moments of time but their opinions do not coincide each other. Formally:

Let $\mathbf{P} \in \mathbb{P}_{N \times T}$ be a time preference profile such that there is at most one agent who has the same opinion at $t_{j}$ and $t_{j+1}$ for $j \in\{1, \ldots T\}$, that is, $n_{0,0}^{t_{j}, t_{j+1}} \leq 1$ and $n_{1,1}^{t_{j}, t_{j+1}} \leq 1$ for all $j \in \mathbf{T}$. Then, $\tau_{S}(\mathbf{P})=0$.

Proof It is immediately from Definition 3.
Leaving minimum time stability: In order to leave the minimum time stability it is needed that at least the opinions of two agents coincide at the same moment of time and the next one. Formally:

Let $\mathbf{P} \in \mathbb{P}_{N \times T}$ be a time preference profile such that there exists at least a $k, k \in \mathbf{T}$, such that $n_{0,0}^{t_{k}, t_{k+1}}>1$ or $n_{1,1}^{t_{k}, t_{k+1}}>1$, then $\tau_{S}(\mathbf{P})>0$.

Proof Using Definition 3 is straightforward.

Time monotonicity: Consider two time preference profiles, $\mathbf{P}$ and $\mathbf{P}^{\prime}$, that coincide in all their elements excepting the opinion of an agent $m \in \mathbf{N}$, at $t_{k}$ and $t_{k+1}$. Concretely, this agent has different opinion at $t_{k}$ and $t_{k+1}$ in $\mathbf{P}: P_{m t_{j}} \neq P_{m t_{j+1}}$, and the agent's opinion is the same at $t_{k}$ and $t_{k+1}$ in $\mathbf{P}^{\prime}: P_{m t_{j}}^{\prime}=P_{m t_{j+1}}^{\prime}$. In this case, the sequential time cohesiveness measure verifies $\tau_{S}\left(\mathbf{P}^{\prime}\right) \geq \tau_{S}(\mathbf{P})$. Formally:

Let $\mathbf{P}, \mathbf{P}^{\prime} \in \mathbb{P}_{N \times T}$ be time preference profiles such that:

a) $P_{i t_{j}}=P_{i t_{j}}^{\prime}, i \in\{\mathbf{N} \backslash\{m\}\}$,

b) $P_{m t_{k}} \neq P_{m t_{k+1}}, m \in \mathbf{N}, t_{k}, t_{k+1} \in \mathbf{T}$,

c) $P_{m t_{k}}^{\prime}=P_{m t_{k+1}}^{\prime}, m \in \mathbf{N}, t_{k}, t_{k+1} \in \mathbf{T}$.

Then, $\tau_{S}\left(\mathbf{P}^{\prime}\right) \geq \tau_{S}(\mathbf{P})$.

Proof It is enough to prove that $\tau_{S}\left(\mathbf{P}^{\prime}\right)-\tau_{S}(\mathbf{P}) \geq 0$. Let $n_{1,1}^{t_{k}, t_{k+1}}$ and $n_{0,0}^{t_{k}, t_{k+1}}$ the number of agents that approve and disapprove alternative $x$ at $t_{k}$ and $t_{k+1}$ from $\mathbf{P}$ and $\left(n_{1,1}^{t_{k}, t_{k+1}}\right)^{\prime}$ and $\left(n_{0,0}^{t_{k}, t_{k+1}}\right)^{\prime}$ the number of agents that approve and disapprove alternative $x$ at $t_{k}$ and $t_{k+1}$ from $\mathbf{P}^{\prime}$.

- If $P_{m t_{k}}^{\prime}=P_{m t_{k+1}}^{\prime}=0$, then

$$
\left(n_{0,0}^{t_{k}, t_{k+1}}\right)^{\prime}=n_{0,0}^{t_{k}, t_{k+1}}+1
$$

and

$$
\begin{aligned}
\tau_{S}\left(\mathbf{P}^{\prime}\right)-\tau_{S}(\mathbf{P}) & = \\
& =\frac{1}{T-1}\left(\frac{\left(n_{0,0}^{t_{k}, t_{k+1}}+1\right)\left(\left(n_{0,0}^{t_{k}, t_{k+1}}+1\right)-1\right)}{N(N-1)}\right) \\
& -\frac{1}{T-1}\left(\frac{n_{0,0}^{t_{k}, t_{k+1}}\left(n_{0,0}^{t_{k}, t_{k+1}}-1\right)}{N(N-1)}\right) \geq 0
\end{aligned}
$$

since for all $z \in \mathbb{N},(z+1) z-z(z-1) \geq 0$.

- If $P_{m t_{k}}^{\prime}=P_{m t_{k+1}}^{\prime}=1$, then

$$
\left(n_{1,1}^{t_{k}, t_{k+1}}\right)^{\prime}=n_{1,1}^{t_{k}, t_{k+1}}+1
$$


and

$$
\begin{aligned}
\tau_{S}\left(\mathbf{P}^{\prime}\right)-\tau_{S}(\mathbf{P}) & = \\
& =\frac{1}{T-1}\left(\frac{\left(n_{1,1}^{t_{k}, t_{k+1}}+1\right)\left(\left(n_{1,1}^{t_{k}, t_{k+1}}+1\right)-1\right)}{N(N-1)}\right) \\
& -\frac{1}{T-1}\left(\frac{n_{1,1}^{t_{k}, t_{k+1}}\left(n_{1,1}^{t_{k}, t_{k+1}}-1\right)}{N(N-1)}\right) \geq 0
\end{aligned}
$$

since for all $z \in \mathbb{N},(z+1) z-z(z-1) \geq 0$.

Convergence to full stability: If new moments of times are repeatedly introduced into the problem and all agents have the same opinion at them, then the sequential time cohesiveness measure approaches 1. Formally:

Suppose that $q$ moments of time $t_{T+1}, \ldots t_{T+q}$ are added to $\mathbf{T}$, and at these new moments of time the alternative $x$ is unanimously approved (resp. unanimously disapproved) by all agents. If the introduction of new moments of time does not affect agents' opinions in past times, then the sequential time cohesiveness measure of the extended time preference profile $\overline{\mathbf{P}}^{(q)} \in \mathbb{P}_{N \times(T+q)}$ approaches 1 when $q$ tends to infinity.

$$
\lim _{q \rightarrow \infty} \tau_{S}\left(\overline{\mathbf{P}}^{(\mathbf{q})}\right)=1
$$

Proof Using time-reducibility,

$$
\begin{aligned}
\tau_{S}\left(\overline{\mathbf{P}}^{(\mathbf{q})}\right) & =\frac{1}{T+q-1} \sum_{j=1}^{T+q-1} \tau_{S}\left(\overline{\mathbf{P}}^{I_{j, j+1}}\right)= \\
& =\frac{1}{T+q-1} \sum_{j=1}^{T} \tau_{S}\left(\overline{\mathbf{P}}^{I_{j, j+1}}\right) \\
& +\frac{1}{T+q-1} \sum_{j=T+1}^{T+q-1} \tau_{S}\left(\overline{\mathbf{P}}^{I_{j, j+1}}\right)= \\
& =\frac{1}{T+q-1} \sum_{j=1}^{T} \tau_{S}\left(\overline{\mathbf{P}}^{I_{j, j+1}}\right) \\
& +\frac{1}{T+q-1} \sum_{j=T+1}^{T+q} 1= \\
& =\frac{1}{T+q-1} \sum_{j=1}^{T} \tau_{S}\left(\overline{\mathbf{P}}^{I_{j, j+1}}\right)+\frac{q-2}{T+q-1}
\end{aligned}
$$

Then when $q$ tends to infinity the first term of $\tau_{S}\left(\overline{\mathbf{P}}^{(\mathbf{q})}\right)$ tends to 0 and the second term tends to 1 .
Convexity: It means the sequential time cohesiveness measure of a time preference profile is a weighted average of the measures of any decomposition of $\mathbf{P}$ into consecutive time-subprofiles. Formally:

For each time preference profile $\mathbf{P} \in \mathbb{P}_{N \times T}$, and each decomposition of $\mathbf{P}$ into two consecutive timesubprofiles, $\mathbf{P}^{I_{1}} \in \mathbb{P}_{N \times\left(k_{1}+1\right)}$ and $\mathbf{P}^{I_{2}} \in \mathbb{P}_{N \times\left(T-k_{1}\right)}$ with $I_{1}=\left\{t_{1}, \ldots, t_{k_{1}+1}\right\}$ and $I_{2}=\left\{t_{k_{1}+1}, \ldots, t_{T}\right\}$, and $\left(\left|I_{1}\right|-1\right)+\left(\left|I_{2}\right|-1\right)=T-1$

$\tau_{S}(\mathbf{P})=\frac{\left(\left|I_{1}\right|-1\right) \cdot \tau_{S}\left(\mathbf{P}^{I_{1}}\right)+\left(\left|I_{2}\right|-1\right) \cdot \tau_{S}\left(\mathbf{P}^{I_{2}}\right)}{T-1}$

Proof It is clear from time-reducibility taking into account the following

$$
\begin{gathered}
\tau_{S}\left(\mathbf{P}^{I_{1}}\right)=\frac{1}{\left|I_{1}\right|-1} \sum_{j=1}^{k_{1}} \tau_{S}\left(\mathbf{P}^{I_{j, j+1}}\right) \\
\tau_{S}\left(\mathbf{P}^{I_{2}}\right)=\frac{1}{\left|I_{2}\right|-1} \sum_{j=k_{1}+1}^{T-1} \tau_{S}\left(\mathbf{P}^{I_{j, j+1}}\right)
\end{gathered}
$$

3 Comparative analysis of preference stability in Clinical Decision Making: The case of terminally cancer patients' last year of life

Since 1991, Patient Self-Determination Acts have become significant with specific regard to life support options [5]. In particular, patients can record their preferences about the type of care that they would like to receive or not in case of loss of decision-making capacity by means an official document called "living will".

In order to collect easily patients' preferences about life support choices, Beland and Froman [5] developed and validated an instrument capable of making patients easy to express their preferences about their options, the Life Support Preferences Questionnaire (LSPQ).

From the LSPQ beginnings to the present, several considerations have increased the significance of such tool. Among these, it can be highlighted the use of the questionnaire like a mechanism to educate patients and their families about the selection of life support choices [30]. In addition, the LSPQ can be used to make efficient and effective health care services at end of life [13] because population ageing are increasingly high health care costs. To tackle the aforementioned aims, it is necessary to achieve a detail study of patients' preferences and their preference stability along their illness.

In consequence, this contribution focuses on studying the stability of preferences for life-sustaining treatments of patients with advanced cancer. To do it, the 
sequential time cohesiveness measure is used taking into account three different treatments and the possibility of cancer metastases.

\subsection{The setting of the study}

So as to implement our proposal for measuring the stability of preferences along time of a group of agents, this contribution is inspired and motivated by the study of Tang et al. [29]. In this contribution, the authors examined the stability of life-sustaining treatment preferences at end of life of cancer patient's last year by means of an statistical approach. They explored longitudinal preference changes based on a sample of 257 patients recruited from March 2009 to December 2012 from the general medical inpatient units of a medical center in Northwest Taiwan and followed up until June, 2013.

Based on this study, a finite set of 257 patients $\mathbf{N}=\{1,2, \ldots, N=257\}$ is considered. Theses patients expressed their opinions on a finite set of 3 treatments for life-sustaining at end of life, $X=\left\{x_{1}, x_{2}, x_{3}\right\}$ being:

- $x_{1}=$ cardiopulmonary resuscitation $(\mathrm{CPR})$,

- $x_{2}=$ dying in an intesive care unit (ICU),

- $x_{3}=$ mechanical ventilation support (MSV).

For that purpose, patients' opinions were collected by means of an interview (an adapted LSPQ) where patients answer questions about their preferences of CPR, ICU and MSV treatment when life was in danger as Figure 1 shows.

In the questionnaire patients expressed their preferences about approving o disapproving the aforementioned treatments at four different time moments along their illness, $\mathbf{T}=\left\{t_{1}, t_{2}, t_{3}, t_{4}\right\}$. To be precise, taking into account patients' time proximity to death:

- $t_{1}=181-365$ days,

- $t_{2}=91-180$ days,

- $t_{3}=31-90$ days,

- $t_{4}=1-30$ days.

Thus, patients' opinions can be formalized by means of a time preference profile for each treatment

$$
\begin{aligned}
\mathbf{P}^{C P R} & =\left(\begin{array}{ccc}
P_{1 t_{1}}^{C P R} & \ldots & P_{1 t_{4}}^{C P R} \\
\vdots & \ddots & \vdots \\
P_{257 t_{1}}^{C P R} & \ldots & P_{257 t_{4}}^{C P R}
\end{array}\right)_{257 \times 4} \\
\mathbf{P}^{I C U} & =\left(\begin{array}{ccc}
P_{1 t_{1}}^{I C U} & \ldots & P_{1 t_{4}}^{I C U} \\
\vdots & \ddots & \vdots \\
P_{257 t_{1}}^{I C U} & \ldots & P_{257 t_{4}}^{I C U}
\end{array}\right)_{257 \times 4}
\end{aligned}
$$

Preferences for life-sustaining treatment questionnaire

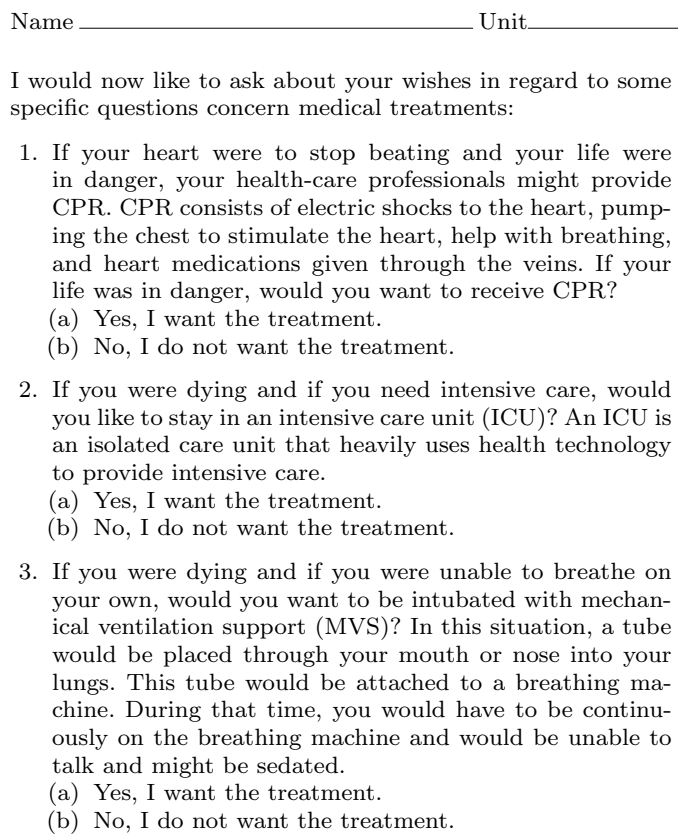

1. If your heart were to stop beating and your life were in danger, your health-care professionals might provide CPR. CPR consists of electric shocks to the heart, pumping the chest to stimulate the heart, help with breathing, and heart medications given through the veins. If your life was in danger, would you want to receive CPR?

(a) Yes, I want the treatment.

(b) No, I do not want the treatment.

2. If you were dying and if you need intensive care, would you like to stay in an intensive care unit (ICU)? An ICU is an isolated care unit that heavily uses health technology to provide intensive care.

(a) Yes, I want the treatment.

(b) No, I do not want the treatment.

3. If you were dying and if you were unable to breathe on your own, would you want to be intubated with mechanical ventilation support (MVS)? In this situation, a tube would be placed through your mouth or nose into your lungs. This tube would be attached to a breathing machine. During that time, you would have to be continuously on the breathing machine and would be unable to talk and might be sedated.

(a) Yes, I want the treatment.

(b) No, I do not want the treatment.

Fig. 1 Adapted LSPQ

$$
\mathbf{P}^{M V S}=\left(\begin{array}{ccc}
P_{1 t_{1}}^{M V S} & \ldots & P_{1 t_{4}}^{M V S} \\
\vdots & \ddots & \vdots \\
P_{257 t_{1}}^{M V S} & \ldots & P_{257 t_{4}}^{M V S}
\end{array}\right)_{257 \times 4}
$$

Suppose the information provided by the three previous time preference profiles can be group in Table 3.

Using Definition 3, the sequential time cohesiveness measure for each profile, that is, for each treatment can be computed. Table 4 shows such values including all moments of time and all patients and Figure 2 displays them.

As it can be seen in Table 4 and Figure 2, there is not much cohesiveness among patients about using lifesustaining treatments at end of live along their illness. The highest value is obtained for CPR treatment.

In order to explore in depth these results, the set of patients is partitioned, distinguish between patients with and without metastases. Table 5 shows the patients' opinions along time taking into account if they have metastases or not.

Taking into account data from Table 5 , the values of the sequential time cohesiveness measure are computed and presented in Table 6 . 


\begin{tabular}{lll} 
Treatment & $n_{0,0}^{t_{1}, t_{2}}$ & $n_{1,1}^{t_{1}, t_{2}}$ \\
\hline CPR & 190 & 34 \\
ICU & 142 & 79 \\
MSV & 170 & 44 \\
\hline
\end{tabular}

\begin{tabular}{lll} 
Treatment & $n_{0,0}^{t_{2}, t_{3}}$ & $n_{1,1}^{t_{2}, t_{3}}$ \\
\hline CPR & 210 & 24 \\
ICU & 156 & 63 \\
MSV & 187 & 38 \\
\hline
\end{tabular}

\begin{tabular}{lll} 
Treatment & $n_{0,0}^{t_{3}, t_{4}}$ & $n_{1,1}^{t_{3}, t_{4}}$ \\
\hline CPR & 228 & 15 \\
ICU & 184 & 26 \\
MSV & 209 & 25 \\
\hline
\end{tabular}

Table 3 Number of patients that approve and disapprove different treatments at different moments of time

\begin{tabular}{lll} 
Treatment & Profile & $\tau_{S}(\mathbf{P})$ \\
\hline CPR & $\mathbf{P}^{C P R}$ & 0.676 \\
ICU & $\mathbf{P}^{I C U}$ & 0.449 \\
MVS & $\mathbf{P}^{M V S}$ & 0.562
\end{tabular}

Table 4 Values of the sequential time cohesiveness measure for each treatment

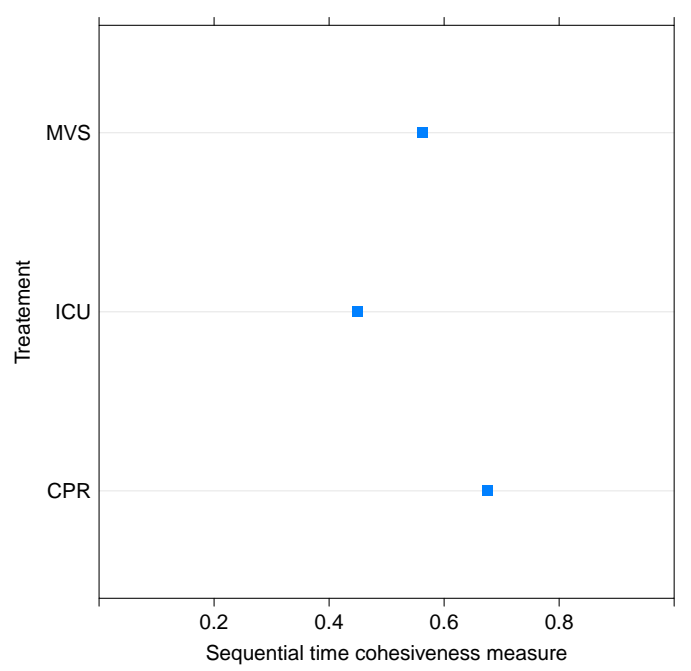

Fig. 2 Graphical display of sequential time cohesiveness measure for the three different treatment calculated from Table 3

Such as it can be observed in Table 6 and Figure 3, the sequential time cohesiveness measure for patients

\begin{tabular}{|c|c|c|c|c|c|c|}
\hline \multirow[b]{2}{*}{ Metastases } & \multicolumn{2}{|c|}{ CPR } & \multicolumn{2}{|c|}{ ICU } & \multicolumn{2}{|c|}{ MVS } \\
\hline & Yes & No & Yes & No & Yes & No \\
\hline$n_{0,0}^{t_{1}, t_{2}}$ & 111 & 79 & 94 & 48 & 98 & 72 \\
\hline$n_{1,1}^{t_{1}, t_{2}}$ & 7 & 27 & 15 & 64 & 5 & 39 \\
\hline$n_{0,0}^{t_{2}, t_{3}}$ & 115 & 95 & 104 & 52 & 112 & 75 \\
\hline$n_{1,1}^{t_{2}, t_{3}}$ & 2 & 22 & 5 & 58 & 4 & 34 \\
\hline$n_{0,0}^{t_{3}, t_{4}}$ & 122 & 106 & 117 & 67 & 119 & 90 \\
\hline$n_{1,1}^{t_{3}, t_{4}}$ & 0 & 15 & 1 & 25 & 1 & 24 \\
\hline
\end{tabular}

Table 5 Number of patients that approve and disapprove the three treatments at different moments of time distinguishing patients with and without metastases

\begin{tabular}{llll} 
Treatment & & Agent-subprofile & $\tau_{S}(\mathbf{P})$ \\
\hline \multirow{2}{*}{ CPR } & Metastases & $\mathbf{P}_{M}^{C P R}$ & 0.863 \\
& No metastases & $\mathbf{P}_{N M}^{C P R}$ & 0.532 \\
\hline \multirow{2}{*}{ ICU } & Metastases & $\mathbf{P}_{M}^{I C U}$ & 0.715 \\
& No metastases & $\mathbf{P}_{N M}^{I C U}$ & 0.333 \\
\hline \multirow{2}{*}{ MVS } & Metastases & $\mathbf{P}_{M}^{M V S}$ & 0.774 \\
& No metastases & $\mathbf{P}_{N M S}^{M V S}$ & 0.421 \\
\hline
\end{tabular}

Table 6 Values of sequential time cohesiveness measure obtained for agent-subprofiles according to metastasis diagnoses

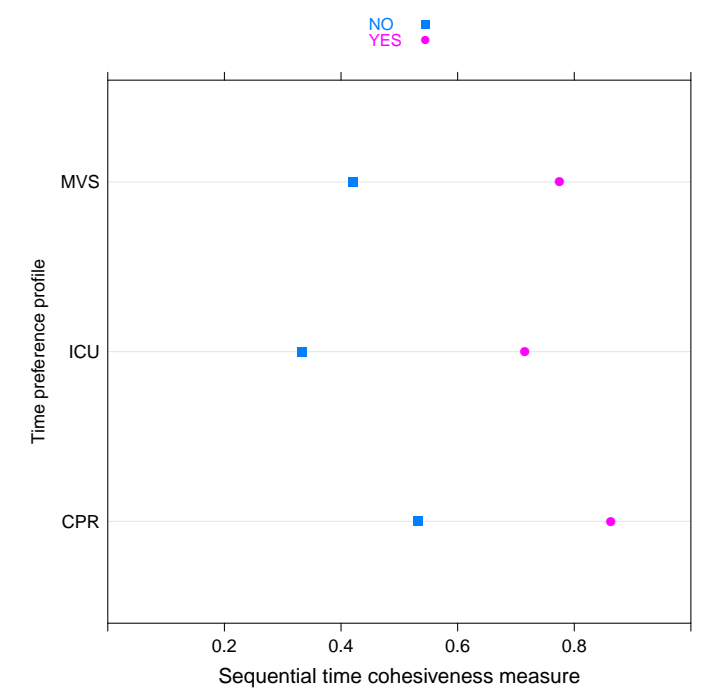

Fig. 3 Graphical display of sequential time cohesiveness measure for the three different treatment taking into account metastases diagnoses calculated from Table 5

suffering metastases is significant greater than the time cohesiveness measure for patients no suffering metastases. This is observed for the three treatments. 
Now regarding the results obtained in Table 6 for CPR treatment, the study is focused on examining in detail the stability of patients' preferences at different time-subprofiles, $\mathbf{P}^{I_{t_{1}, t_{2}}}, \mathbf{P}^{I_{t_{2}, t_{3}}}, \mathbf{P}^{I_{t_{3}, t_{4}}}$, and considering two agent-subprofiles $\mathbf{P}_{M}$ and $\mathbf{P}_{N M}$.

Table 7 presents the number of agents that approve or disapprove CPR at different moments of time. From Table 7, the sequential cohesiveness measure is computed for the aforementioned time-subprofiles (see Table 8 ). Tables 9 and 10 show the values of the sequential time cohesiveness measure for the time-subprofiles distinguishing patients with and without metastases. Figure 4 represents values from Tables 8, 9 and 10 .

\begin{tabular}{l|rr|r}
$t_{1}$ & No & Yes & \\
\hline No & $n_{0,0}^{t_{1}, t_{2}}=190$ & $n_{0,1}^{t_{1}, t_{2}}=8$ & $n_{0}^{t_{1}}=198$ \\
Yes & $n_{1,0}^{t_{1}, t_{2}}=25$ & $n_{1,1}^{t_{1}, t_{2}}=34$ & $n_{1}^{t_{1}}=59$ \\
\hline & $n_{0}^{t_{2}}=215$ & $n_{1}^{t_{2}}=42$ & $N=257$
\end{tabular}

\begin{tabular}{l|rr|r}
$t_{2}$ & No & Yes & \\
\hline No & $n_{0,0}^{t_{2}, t_{3}}=210$ & $n_{0,1}^{t_{2}, t_{3}}=5$ & $n_{0}^{t_{2}}=215$ \\
Yes & $n_{1,0}^{t_{2}, t_{3}}=18$ & $n_{1,1}^{t_{2}, t_{3}}=24$ & $n_{1}^{t_{2}}=42$ \\
\hline & $n_{0}^{t_{3}}=228$ & $n_{1}^{t_{3}}=29$ & $N=257$
\end{tabular}

\begin{tabular}{l|rr|r}
\multicolumn{1}{c|}{$t_{3}$} & No & Yes & \\
\hline No & $n_{0,0}^{t_{3}, t_{4}}=228$ & $n_{0,1}^{t_{3}, t_{4}}=0$ & $n_{0}^{t_{3}}=228$ \\
Yes & $n_{1,0}^{t_{3}, t_{4}}=14$ & $n_{1,1}^{t_{3}, t_{4}}=15$ & $n_{1}^{t_{3}}=29$ \\
\hline & $n_{0}^{t_{4}}=242$ & $n_{1}^{t_{4}}=15$ & $N=257$
\end{tabular}

Table 7 Number of agents that approve or disapprove CPR at different moments of time

\begin{tabular}{lll} 
Treatment & Time-subprofile & $\tau_{S}(\mathbf{P})$ \\
\hline CPR & $\mathbf{P}^{I_{t_{1}, t_{2}} C P R}$ & 0.563 \\
& $\mathbf{P}^{I_{t_{2}, t_{3}} C P R}$ & 0.675 \\
& $\mathbf{P}^{I_{t_{3}, t_{4}} C P R}$ & 0.790
\end{tabular}

Table 8 Values of sequential time cohesiveness measures for CPR according to different time-subprofiles

To conclude, it can be observed that preferences of patients with metastases are the most stable considering all moments of time and also for each timesubprofile.

\begin{tabular}{lll} 
Treatment & Time-subprofile & $\tau_{S}(\mathbf{P})$ \\
\hline CPR & $\mathbf{P}_{M}^{I_{t_{1}, t_{2}} C P R}$ & 0.790 \\
& $\mathbf{P}_{M}^{I_{t_{2}, t_{3}} C P R}$ & 0.846 \\
& $\mathbf{P}_{M}^{I_{t_{3}, t_{4}} C P R}$ & 0.952 \\
\hline
\end{tabular}

Table 9 Values of sequential time cohesiveness measures for CPR according to different time-subprofiles and for agentsubprofile of patients with metastases diagnoses

\begin{tabular}{lll} 
Treatment & Time-subprofile & $\tau_{S}(\mathbf{P})$ \\
\hline CPR & $\mathbf{P}_{N M}^{I_{t_{1}, t_{2}} C P R}$ & 0.397 \\
& $\mathbf{P}_{N M}^{I_{t_{2}, t_{3}} C P R}$ & 0.543 \\
& $\mathbf{P}_{N M}^{I_{t_{3}, t_{4}} C P R}$ & 0.656 \\
\hline
\end{tabular}

Table 10 Values of sequential time cohesiveness measures for CPR according to different time-subprofiles and for agentsubprofile of patients without metastases diagnoses

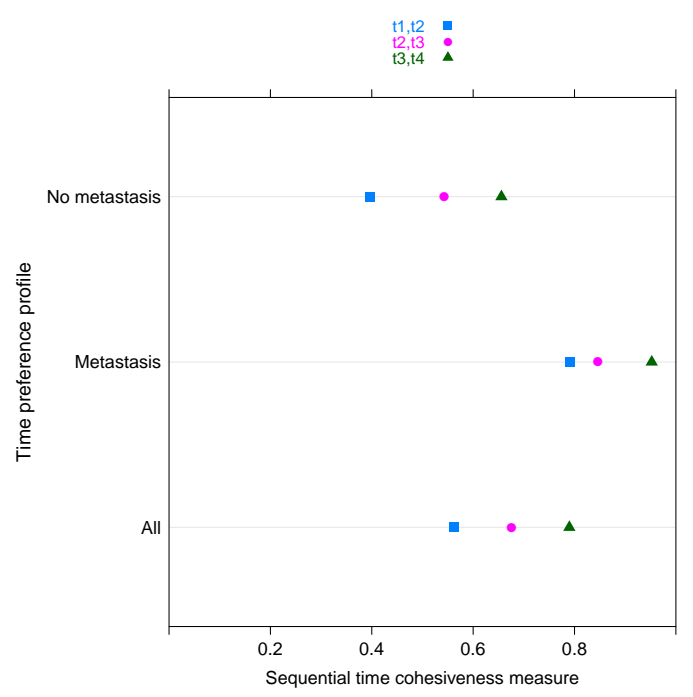

Fig. 4 Graphical display of sequential time cohesiveness measures for CPR, time-subprofiles and agent-subprofiles calculated from Tables 8,9 and 10

\section{Concluding remarks}

Research on preference stability topic has advanced mainly in Economics. In this work, a non-traditional perspective is set out. The problem of measuring the degree of cohesiveness in a setting where agents express their opinions on an alternative at different times by means of an approval or disapproval evaluation is explored. A general concept of time cohesiveness measure is introduced and a particular formulation based on the consideration of any two successive times is proposed, namely the sequential time cohesiveness measure. Some properties which make our proposal appealing are also 
provided. Those properties are common in traditional consensus measures.

The applicability of our proposal to real situations is emphasized by means of adapting a factual problem in Clinical Decision Making. Concretely, the case of terminally cancer patients' last year of life is studied using the new sequential time cohesiveness measure.

Some straight lines of future research that could be addressed from the new approach are listed bellow:

- It could be interesting to analyse preference stability problem and its measure when the number of experts decreases along time because loss of experts to follow-up e.g., patients deaths before ended study.

- In some cases, experts could not be capable of expressing their opinion about an alternative, that is, they are undecided on it. Under this assumption it could be appealing to develop a specific time cohesiveness measure.

- Many problems from a diversity of fields could be tackled such as the consumers' preferences, Clinical Decision Making problems and so on.

Acknowledgements The authors thank the anonymous reviewers and S. García López and F. Herrera (Editors-in-Chief) for their valuable comments and recommendations. The authors acknowledge financial support by the Spanish Ministerio de Ciencia e Innovación under Project Project ECO201677900-P.

\section{References}

1. Alcalde-Unzu, J., Vorsatz, M.: Do we agree? Measuring the cohesiveness of preferences. Theory and Decision 80, 313-339 (2016)

2. Alcantud, J., de Andrés Calle, R., Cascón, J.: On measures of cohesiveness under dichotomous opinions: Some characterizations of approval consensus measures. Information Sciences 240, 45-55 (2013)

3. Alcantud, J., de Andrés Calle, R., Cascón, J.: Pairwise dichotomous cohesiveness measures. Group Decision and Negotiation 24(5), 833-854 (2015)

4. Anderson, L., Mellor, J.: Are risk preferences stable? Comparing an experimental measure with a validated survey-based measure. Journal of Risk and Uncertainty 39(2), 137-160 (2009)

5. Beland, D.K., Froman, R.D.: Preliminary validation of a measure of life support preferences. Journal of Nursing Scholarship 27(4), 307-310 (1995)

6. Bosch, R.: Characterizations of voting rules and consensus measures. Ph.D. thesis, Tilburg University (2005)

7. Brandts, J., Charness, G.: Hot vs. cold: Sequential responses and preference stability in experimental games. Experimental Economics 2(3), 227-238 (2000)

8. Brouwer, R.: Constructed preference stability: a testretest. Journal of Environmental Economics and Policy 1(1), 70-84 (2012)
9. Cameron, L., Shah, M.: Risk-taking behavior in the wake of natural disasters. Journal of Human Resources $\mathbf{5 0}(2)$, 484-515 (2015)

10. Carlsson, F., Johansson-Stenman, O., Nam, P.: Social preferences are stable over long periods of time. Journal of Public Economics 117, 104-114 (2014)

11. Chuang, Y., Schechter, L.: Stability of experimental and survey measures of risk, time, and social preferences: A review and some new results. Journal of Development Economics 117, 151 - 170 (2015)

12. Doyle, J.: Survey of time preference, delay discounting models. Judgment and Decision Making 8(2), 116-135 (2013)

13. Finkelstein, E.A., Bilger, M., Flynn, T.N., Malhotra, C.: Preferences for end-of-life care among communitydwelling older adults and patients with advanced cancer: A discrete choice experiment. Health Policy 119(11), $1482-1489$

14. García-Lapresta, J.L., Pérez-Román, D.: Measuring consensus in weak orders. In: E. Herrera-Viedma, J.L. García-Lapresta, J. Kacprzyk, M. Fedrizzi, H. Nurmi, S. Zadrozny (eds.) Consensual Processes, Studies in Fuzziness and Soft Computing, vol. 267, pp. 213-234. Springer Berlin Heidelberg (2011)

15. Gerrans, P., Faff, R., Hartnett, N.: Individual financial risk tolerance and the global financial crisis. Accounting and Finance 55(1), 165-185 (2015)

16. González-Arteaga, T., Alcantud, J., de Andrés Calle, R.: A cardinal dissensus measure based on the Mahalanobis distance. European Journal of Operational Research 251(2), 575 - 585 (2016)

17. González-Pachón, J., Romero, C.: Aggregation of ordinal and cardinal preferences: a framework based on distance functions. Journal of Multi-Criteria Decision Analysis 15(3-4), 79-85 (2009)

18. González-Pachón, J., Romero, C., Díaz-Balteiro, L.: How to combine inconsistent ordinal and cardinal preferences: a satisficing modelling approach. Computers and Industrial Engineering 67, 168-172 (2014)

19. Herrera-Viedma, E., Herrera, F., Chiclana, F.: A consensus model for multiperson decision making with different preference structures. IEEE Transactions on Systems, Man, and Cybernetics - Part A: Systems and Humans 32(3), 394-402 (2002)

20. Hoeffler, S., Ariely, D.: Constructing stable preferences: A look into dimensions of experience and their impact on preference stability. Journal of Consumer Psychology 8(2), 113-139 (1999)

21. Jakiela, P., Miguel, E., te Velde, V.: You've earned it: estimating the impact of human capital on social preferences. Experimental Economics 18(3), 385-407 (2015)

22. Krupka, E., Stephens, M.: The stability of measured time preferences. Journal of Economic Behavior and Organization 85(1), 11-19 (2013)

23. Loewenstein, G., Angner, E.: Predicting and indulging changing preferences. Time and decision: Economic and psychological perspectives on intertemporal choice pp. 351-391 (2003)

24. Meier, S., Sprenger, C.: Temporal stability of time preferences. Review of Economics and Statistics 97(2), 273-286 (2015)

25. Necker, S., Ziegelmeyer, M.: Household risk taking after the financial crisis. The Quarterly Review of Economics and Finance 59, 141 - 160 (2016)

26. Reynaud, A., Couture, S.: Stability of risk preference measures: Results from a field experiment on french farmers. Theory and Decision 73(2), 203-221 (2012) 
27. Sahm, C.: How much does risk tolerance change? Quarterly Journal of Finance 02(04) (2012)

28. Shen, A., Ball, A.: Preference stability belief as a determinant of response to personalized recommendations. Journal of Consumer Behaviour 10(2), 71-79 (2011)

29. Tang, S.T., Wen, F.H., Hsieh, C.H., Chou, W.C., Chang, W.C., Chen, J.S., Chiang, M.C.: Preferences for lifesustaining treatments and associations with accurate prognostic awareness and depressive symptoms in terminally ill cancer patients' last year of life. Journal of Pain and Symptom Management 51(1), 41-51 (2016)

30. Winter, L., Parker, B.: Current health and preferences for life-prolonging treatments: An application of prospect theory to end-of-life decision making. Social Science \& Medicine 65(8), 1695 - 1707 (2007) 